\title{
Photometric classification of Supernovae from the SUDARE survey
}

\author{
Giuliano Pignata \\ Departamento de Ciencias Fisicas, Universidad Andres Bello, \\ Avenida Republica 252, Santiago, Chile \\ email: gpignata@unab.cl
}

\begin{abstract}
I present first results from the algorithm we developed to photometrically classify the Supernovae discovered by the Supernova Diversity and Rate Evolution (SUDARE). The classification outcome is compared with that of the public available code SNANA.
\end{abstract}

Keywords. supernovae: general, methods: statistical, surveys

\section{Introduction}

SUDARE is a survey which main goal is discover Supernovae (SNe) of all types in the redshift range $\mathrm{z}=0.3-0.6$, where the rate of Core Collapse (CC) SNe is basically unexplored and the published estimates of the SN Ia rate show some discrepancy. The properties of the SNe discovered will be compared with those of the local sample and linked to the properties of the parent stellar populations. For the latter we choose to monitor the Cosmic Evolution Survey (COSMOS) and Chandra Deep Field South (CDFS) fields. These fields have got an unrivalled coverage from the X-Ray to the Radio. This outstanding data-sets have allowed, among other high-quality data products, a very precise photometric redshifts which are used as a input in our photometric classification algorithm. SUDARE is a rolling-search carried out using the one square degree field of view OMEGACAM camera mounted on the 2.6 meters VLT Survey Telescope (VST) located at ESO Paranal Observatory. To construct a detailed light curve necessary for the photometric classification and to further investigate the SN diversity, we monitor the selected fields in $r$ band with the frequency of one exposure every three days at a limiting magnitude $\mathrm{r}=24.5(\mathrm{~S} / \mathrm{N}=5.0)$ and once every week, we are obtaining $g$ and $i$ bands exposures of similar depth. In the images collected on the CDFS and COSMOS field during the first two seasons, we discovered 113 bona-fide SNe.

\section{Photometric classification}

To classify our discoveries, we developed a simple procedure which assess whether the transient multi-colour light curves match a known SN type and what are the best match sub-type, redshift and extinction. To this aim we selected a sample of SN templates of different types for which both multi-colour light curves and spectral sequence are available. The latter are needed to have an estimate of the K-correction. The templates were selected to represent the well established SN types, namely Ia, Ib, Ic, IIb, II and IIn. We also included some templates of the recently discovered class of very bright SNe (e.g. Gal-Yam (2012)) that, although intrinsically very rare Quimby et al. (2013), may easily be detected even at very high redshift.

For all templates we constructed a grid of light curves in apparent magnitudes for each of the survey filters assuming redshifts in the range $0.0<z<1.0$ and extinction 

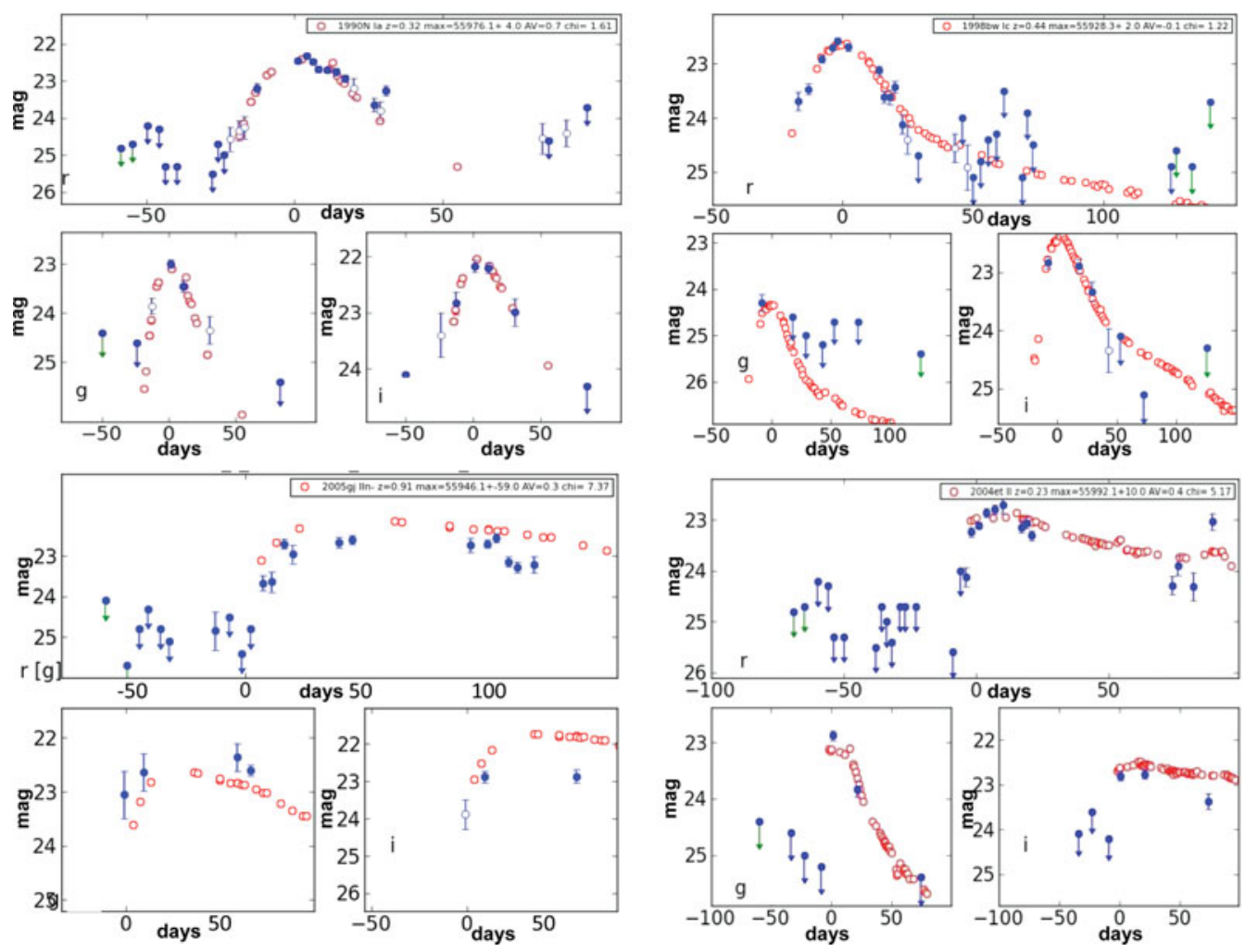

Figure 1. Examples of photometric classifications we obtained using our code. Each quadrant consist of three boxes corresponding to the $r$ band (top), $g$ band (bottom left) and $i$ band (bottom right). Blue points (filled and open) are the SUDARE measured magnitude. Open red points are the template light curves. In the label of the $r$ band box are reported the best match template, the SN type, the redshift, the epoch of the $V$ band maximum light, the extinction in the $V$ band and the value of the reduced $\chi^{2}$.

$0.5<A_{V}<2.0 \mathrm{mag}$. Also the epoch of maximum is allowed to range \pm 100 days from the epoch of the candidate brightest magnitude. We then compare the candidate light curve in each filter with all simulated light curves of the grid. Through $\chi^{2}$ minimization we derive the SN type, average distance and reddening combining measurement for all filters weighting for the number of observed epochs. In case the host galaxy redshift is known, either from spectroscopic or photometric redshift, the distance range is restricted to the measurement error, making the classification precess much faster. Examples of our classification output are reported in Figure 1. We also photometrically classify our discovery using the public available code SNANA (SuperNova ANAlysis) Kessler et al. (2009). The comparison between the results obtained by the two algorithms are shown in the pie chart reported in Figure 2. In this case we collapsed our six SN types in three groups namely: type Ia, Ibc and II. As it is visible there is a good agreement between the output of the two codes. Indeed $86 \%$ of the Type Ia SNe classified by our code were confirmed by SNANA. The remaining objects were classified as Type II (5\%), Ibc (4\%) and for 5\% SNANA did not provide a SN classification.

We found a slightly poorer agreement for the type II classification: $74 \%$ were confirmed by SNANA, while $11 \%$ were classified by the same code as Ia and $4 \%$ as Ibc. For the remaining $11 \%$ SNANA did not provide a SN classification. Some of these objects could be AGN which nature will be definitely established during the survey progress. Finally, we 


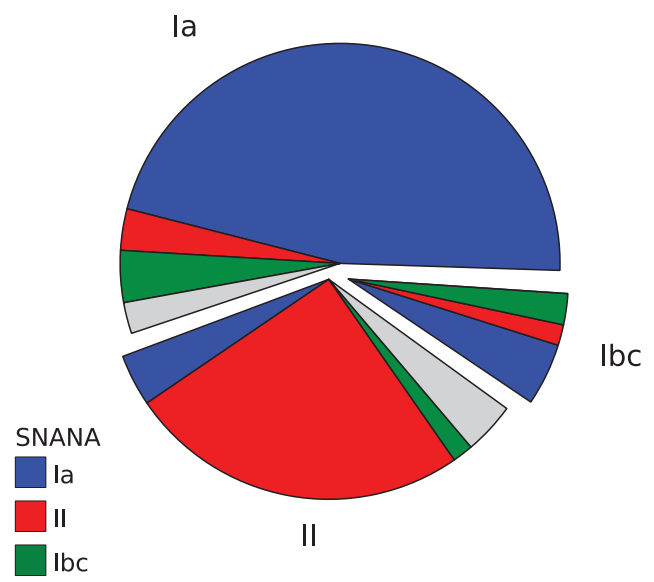

Figure 2. Comparison between the classification obtained using our classifier and SNANA. See text for details.

found the worse agreement in the case of type Ibc SNe were only $50 \%$ of our classification was confirmed by SNANA. Considering the reduced numbers of such objects is premature to draw any statistically meaningful conclusion about the latter discrepancy.

\section{Future work}

For the future we plan to explore more sophisticated techniques for the SN photometric classification of the SUDARE transient starting with the Kernel Principal Component Analysis method (see Ishida \& de Souza (2013)) combined with machine learning algorithms.

\section{References}

Gal-Yam, A. 2012, Science, 337, 927

Kessler, R., Bernstein, J. P., Cinabro, D., Dilday, B., Frieman, J. A., Jha, S., Kuhlmann, S., Miknaitis, G., Sako, M., Taylor, M., \& Vanderplas, J. 2009, PASP, 121, 883

Ishida, E. E. O. \& de Souza R. S. 2013, MNRAS, 430, 509

Quimby, R. M., Yuan, F., Akerlof, C., \& Wheeler, J. C. 2013, MNRAS, 431, 912 